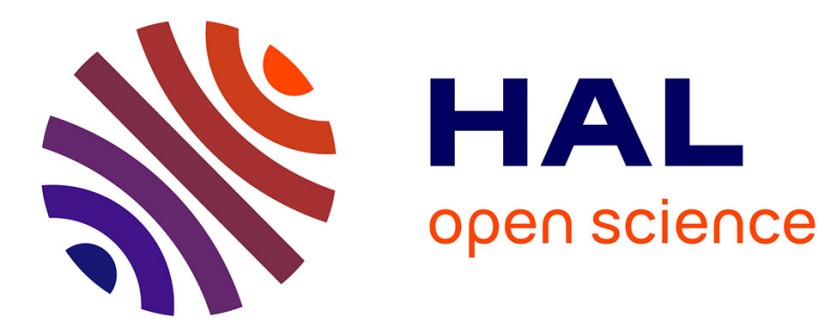

\title{
Towards the Robust Intersection of Implicit Quadrics
}

\author{
Laurent Dupont, Daniel Lazard, Sylvain Lazard, Sylvain Petitjean
}

\section{To cite this version:}

Laurent Dupont, Daniel Lazard, Sylvain Lazard, Sylvain Petitjean. Towards the Robust Intersection of Implicit Quadrics. J. Winkler and M. Niranjan. Uncertainty in Geometric Computations, Kluwer Academic Publishers, pp.59-68, 2002, International Series in Engineering and Computer Science. inria-00100878

\section{HAL Id: inria-00100878 https://hal.inria.fr/inria-00100878}

Submitted on 15 Dec 2009

HAL is a multi-disciplinary open access archive for the deposit and dissemination of scientific research documents, whether they are published or not. The documents may come from teaching and research institutions in France or abroad, or from public or private research centers.
L'archive ouverte pluridisciplinaire HAL, est destinée au dépôt et à la diffusion de documents scientifiques de niveau recherche, publiés ou non, émanant des établissements d'enseignement et de recherche français ou étrangers, des laboratoires publics ou privés. 


\title{
Chapter 1
}

\section{TOWARDS THE ROBUST INTERSECTION OF IMPLICIT QUADRICS}

\author{
Laurent Dupont, Sylvain Lazard and Sylvain Petitjean \\ Loria-CNRS 83 Inria Lorraine \\ BP 239, 54506 Vandouvre cedex, France \\ \{dupont,lazard, petitjea\} @loria.fr \\ Daniel Lazard \\ LIP6, Université Pierre et Marie Curie \\ Boîte 168, 4 place Jussieu, 75252 Paris cedex 05, France \\ Daniel.Lazard@lip6.fr
}

\begin{abstract}
We are interested in efficiently and robustly computing a parametric form of the intersection of two implicit quadrics with rational coefficients. Our method is similar in spirit to the general method introduced by J. Levin for computing an explicit representation of the intersection of two quadrics, but extends it in several directions. Combining results from the theory of quadratic forms, a projective formalism and new theorems characterizing the intersection of two quadratic surfaces, we show how to obtain parametric representations that are both "simple" (the size of the coefficients is small) and "as rational as possible".
\end{abstract}

Keywords: Robustness of geometric computations, quadric surface intersection.

\section{Introduction}

Computing the intersection of quadric surfaces is an important step in the boundary evaluation of second-order CSG solids [13] and in the determination of the convex hull of quadric surface patches [7].

In solid modeling, the two most widely used types of object representation are constructive solid geometry (CSG) and boundary representation (B-Rep). Since both representations have their own respec- 
tive advantages (modeling is more flexible and intuitive with primitive solids, but rendering on graphical display systems is easier with B-Reps), solid modeling kernels often need an efficient and reliable way to switch from CSG to B-Rep representation. Boundary evaluation, also known as CSG-to-B-Rep conversion, is a well understood problem. However, past approaches have often put more emphasis on efficiency than on robustness and accuracy. Most current modelers use only finite-precision arithmetic for CSG-to-B-Rep conversion. The topological consistency of the computed B-Rep can easily be jeopardized by small amounts of error in the data introduced by finite-precision computations. For many applications in design and automated manufacturing, where topological consistency and possibly accuracy are critical, this may be unacceptable.

Designing reliable, accurate and robust algorithms is currently a major interest of the computational geometry and solid modeling research communities (see, e.g., [2, 14]). A number of approaches have been proposed for the robust and accurate boundary evaluation of polyhedral models $[1,4]$. Most rely heavily on numerical computation, with varying dependence on exact and floating-point arithmetic. Computing the topological structure of a B-Rep involves accurate evaluation of signs of arithmetic expressions. Assuming the input data has a bounded precision and allowing whatever bit-length is necessary for number representation, these signs can be computed exactly.

By contrast, there has been much less work on robust CSG-to-B-Rep conversion algorithms for curved primitives. A major reason is that outside the linear realm, exact arithmetic computations require algebraic numbers which cannot in general be represented explicitly with a finite number of bits. In addition, computation with algebraic numbers is extremely slow. One notable exception is the work of Keyser et al. [8, 9] on the boundary evaluation of low-degree CSG solids specified with rational parametric surfaces. The authors use exact arithmetic, present compact data structures for representing the boundary curves as algebraic curves and the boundary vertices as algebraic numbers and use efficient algorithms for computing the intersection curves of parametric surfaces.

The quadratic nature of the equations defining quadric surfaces permits an explicit representation of the intersection curves. In other words, it is theoretically possible to compute a fully parametric representation of the boundary of quadric-based solids. The general method for computing an explicit parametric representation of the intersection between two quadrics is due to J. Levin $[11,12]$. This seminal work has been extended in many different directions. For instance, arguing that Levin's method does not yield explicit information on the morphological type 
of the intersection curve, Farouki et al. [3] made a complete theoretical study, for general quadric surfaces, of degenerate cases. Goldman and Miller [6] took a different path and developed a special-case solution for each of the possible pairs of natural quadrics (i.e., planes, right cones, circular cylinders and spheres).

Most of these methods were motivated by the belief that general methods for intersecting implicit quadric surfaces may not be numerically robust and may fail in degenerate configurations. Indeed, no one has reported thus far an algorithm for robustly computing the intersection between two general quadric surfaces. Recently, however, Geismann et al. [5] have shown how to exactly compute a cell in an arrangement of three quadrics.

We show in this paper that the method of Levin can be improved in several ways in order to remove most of the sources of numerical instabilities in the original algorithm. Using a combination of projective formalism, reduction of quadratic forms and new theorems characterizing the intersection of some quadric surfaces, we show, in particular, how to avoid the appearance of nested radicals (high-degree algebraic numbers).

We present in Section 2 basic definitions and notation. We recall in Section 3 Levin's method for intersecting two quadric surfaces, and present our method in Section 4.

\section{Preliminaries}

In the rest of this paper, we assume that each quadric surface $\mathcal{P}$ is given as the zero-set in $\mathbb{R}^{3}$ of a quadratic implicit equation $P$ with rational coefficients in the variables $\left(x_{1}, x_{2}, x_{3}\right)$, i.e.,

$$
\begin{aligned}
\alpha_{1} x_{1}^{2}+\alpha_{2} x_{2}^{2}+\alpha_{3} x_{3}^{2}+2 \alpha_{4} x_{1} x_{2}+2 \alpha_{5} x_{1} x_{3}+2 \alpha_{6} x_{2} x_{3} \\
+2 \alpha_{7} x_{1}+2 \alpha_{8} x_{2}+2 \alpha_{9} x_{3}+\alpha_{10}=0
\end{aligned}
$$

with $\alpha_{i} \in \mathbb{Q}, i=1, \ldots, 10$ and some $\alpha_{i} \neq 0$. Eq. (1.1) can be rewritten in matrix form as $\mathbf{X}^{T} P \mathbf{X}=0$, with $\mathbf{X}=\left(x_{1}, x_{2}, x_{3}, 1\right)^{T}$ and $P$ the symmetric $4 \times 4$ matrix

$$
P=\left(\begin{array}{cccc}
\alpha_{1} & \alpha_{4} & \alpha_{5} & \alpha_{7} \\
\alpha_{4} & \alpha_{2} & \alpha_{6} & \alpha_{8} \\
\alpha_{5} & \alpha_{6} & \alpha_{3} & \alpha_{9} \\
\alpha_{7} & \alpha_{8} & \alpha_{9} & \alpha_{10}
\end{array}\right)
$$

We use the same notation $P$ for a quadratic implicit equation and its associated matrix; the corresponding quadric surface is denoted $\mathcal{P}$. 
A quadric is said to be given in canonical form if its equation in some coordinate frame is of the form

$$
\begin{aligned}
& \sum_{i=1}^{p} a_{i} x_{i}^{2}-\sum_{i=p+1}^{r} a_{i} x_{i}^{2}+\xi=0 \text { or } \\
& \sum_{i=1}^{p} a_{i} x_{i}^{2}-\sum_{i=p+1}^{r} a_{i} x_{i}^{2}-x_{r+1}=0,
\end{aligned}
$$

with $a_{i}>0, \forall i, \xi \in\{0,1\}$ and $p \leqslant r$.

The signature of $P$ is an ordered pair $(p, q)$ where $p$ and $q$ are the numbers of positive and negative eigenvalues of $P$, respectively (the number of null eigenvalues thus follows from the signature). If $P$ has signature $(p, q)$ then $-P$ has signature $(q, p)$, however, the quadric surfaces associated with $P$ and $-P$ are identical. We thus define here the signature of a quadric surface $\mathcal{P}$ as the couple $(p, q), p \geqslant q$, where $p$ and $q$ are the numbers of positive and negative eigenvalues of $P$ (non-respectively).

We will refer to the $3 \times 3$ upper left submatrix of $P$, denoted $P_{u}$, as the principal submatrix of $P$. The determinant of this matrix is called the principal subdeterminant.

Given two quadrics $\mathcal{P}$ and $\mathcal{Q}$, the pencil generated by $\mathcal{P}$ and $\mathcal{Q}$ is the set of quadrics $\mathcal{R}(\lambda, \mu)$ of equation $\lambda P+\mu Q$ where $(\lambda, \mu) \in \mathbb{R}^{2} \backslash\{0,0\}$. For simplicity of notation, we will consider instead the pencil generated by $\mathcal{P}$ and $\mathcal{Q}$ as the set of quadrics $\mathcal{R}(\lambda)$ of equation $P-\lambda Q, \lambda \in \mathbb{R}$, augmented by the matrix $\mathcal{Q}$. For simplicity of presentation, we will write that the quadric $\mathcal{R}(\lambda)$ is equal to $\mathcal{P}-\lambda \mathcal{Q}$. Finally, recall the wellknown result that the intersection between two distinct quadric surfaces in a pencil does not depend on the choice of the two quadrics.

\section{Levin's method}

We describe in this section the method presented by J. Levin in [11, 12] for computing a parameterized expression for the intersection of two quadric surfaces given by their implicit equations.

Assume that $\mathcal{P}$ and $\mathcal{Q}$ are two distinct quadric surfaces. Levin's method is based on his following key result.

Theorem 1 ([11]) The pencil generated by two distinct quadric surfaces contains at least one simple ruled quadric, i.e., the empty set or a quadric listed in Table 1.1.

One important property of simple ruled quadrics is that the value of their principal sub-determinants is zero. Levin's method is as follows.

1 Find a simple ruled quadric in the pencil generated by $\mathcal{P}$ and $\mathcal{Q}$.

This is achieved by computing the type of $\mathcal{Q}$ and of the quadrics 
Table 1.1. Parameterization of canonical simple ruled quadrics

\begin{tabular}{ccc}
\hline quadric & canonical equation & parameterization \\
& $a_{i}>0$ & $\mathbf{X}=\left[x_{1}, x_{2}, x_{3}\right], u, v \in \mathbb{R}$ \\
\hline line & $a_{1} x_{1}^{2}+a_{2} x_{2}^{2}=0$ & $\mathbf{X}(u)=[0,0, u]$ \\
simple plane & $x_{1}=0$ & $\mathbf{X}(u, v)=[0, u, v]$ \\
double plane & $a_{1} x_{1}^{2}=0$ & $\mathbf{X}(u, v)=[0, u, v]$ \\
parallel planes & $a_{1} x_{1}^{2}=1$ & $\mathbf{X}(u, v)=\left[\frac{1}{\sqrt{a_{1}}}, u, v\right]$, \\
& & $\mathbf{X}(u, v)=\left[-\frac{1}{\sqrt{a_{1}}}, u, v\right]$ \\
intersecting planes & $a_{1} x_{1}^{2}-a_{2} x_{2}^{2}=0$ & $\mathbf{X}(u, v)=\left[\frac{u}{\sqrt{a_{1}}}, \frac{u}{\sqrt{a_{2}}}, v\right]$, \\
hyperbolic paraboloid & $a_{1} x_{1}^{2}-a_{2} x_{2}^{2}-x_{3}=0$ & $\mathbf{X}(u, v)=\left[\frac{u}{\sqrt{a_{1}}},-\frac{u}{\sqrt{a_{2}}}, v\right]$ \\
parabolic cylinder & $a_{1} x_{1}^{2}-x_{2}=0$ & $\mathbf{X}(u, v)=\left[\frac{u+v}{2 \sqrt{a_{1}}}, \frac{u-v}{2 \sqrt{a_{2}}}, u v\right]$ \\
hyperbolic cylinder & $a_{1} x_{1}^{2}-a_{2} x_{2}^{2}=1$ & $\mathbf{X}(u, v)=\left[u, a_{1} u^{2}, v\right]$ \\
& & $\mathbf{X}(u, v)=\left[\frac{1}{2 \sqrt{a_{1}}}\left(u+\frac{1}{u}\right)\right.$, \\
$\frac{2 \sqrt{a_{2}}}{u}$ & & \\
\hline
\end{tabular}

$\mathcal{R}(\lambda)=\mathcal{P}-\lambda \mathcal{Q}$ such that $\lambda$ is solution ${ }^{1}$ of $\operatorname{det}\left(R_{u}(\lambda)\right)=0$. By Theorem 1, one of these quadric surfaces is simple ruled. Let $\mathcal{R}$ be such a quadric and assume that $\mathcal{R}$ and $\mathcal{P}$ are distinct (otherwise, choose $\mathcal{Q}$ instead of $\mathcal{P}$ ).

2 Compute the orthogonal transformation $\mathcal{T}$ which sends $\mathcal{R}$ into canonical form. In the orthonormal coordinate frame in which $\mathcal{R}$ is canonical, $\mathcal{R}$ has one of the parameterizations $\mathbf{X}$ of Table 1.1. Compute the matrix $P^{\prime}=\mathcal{T}^{-1} P \mathcal{T}$ of the quadric surface $\mathcal{P}$ in the canonical frame of $\mathcal{R}$ and consider the equation (augment $\mathbf{X}$ by a fourth coordinate set to 1$)$

$$
\mathbf{X}^{T} P^{\prime} \mathbf{X}=a(u) v^{2}+b(u) v+c(u)=0 .
$$

The parameterizations of Table 1.1 are such that $a(u), b(u)$ and $c(u)$ are polynomials of degree two in $u$.

3 Solve (1.3) for $v$ in terms of $u$ and determine the corresponding domain of validity of $u$ on which the solutions are defined. This domain can be computed exactly since it is the set of $u$ for which the polynomial of degree four $\Delta(u)=b^{2}(u)-4 a(u) c(u) \geqslant 0$. Substituting $v$ by its expression in terms of $u$ in $\mathbf{X}$, we have a parameterization of $\mathcal{P} \cap \mathcal{Q}=$ $\mathcal{P} \cap \mathcal{R}$ in the orthonormal coordinate system where $\mathcal{R}$ is canonical.

4 Report $\mathcal{T} \mathbf{X}(u)$ and the corresponding domain of $u \in \mathbb{R}$ on which the solution is defined, as the parameterized equation of $\mathcal{P} \cap \mathcal{Q}$ in the global coordinate frame.

Levin's method is very nice and powerful since it gives an explicit parametric expression for the intersection of two quadric surfaces given by 
their implicit equations. However, in terms of precision and robustness, the method is not ideal because it introduces many irrational numbers. Thus, if a floating point representation of numbers is used, the result is imprecise or worse, the implementation crashes if no simple ruled quadric is found in step one. If an exact arithmetic representation is used, then the computations are slow because of the high degree of the algebraic numbers; in practice however, because of the high degree of these algebraic numbers, a correct implementation using exact arithmetic seems fairly impossible.

To be precise, the method introduces irrational numbers (namely square and cubic roots) at the following different steps:

- In Step $1, \lambda$ is the root of a degree 3 polynomial in $\mathbb{Q}[X] ; \lambda$ can thus be expressed with two levels of nested radicals (cubic and square roots).

- In Step 2, the coefficients of the transformation matrix $\mathcal{T}$ are expressed with 4 levels of nested roots; indeed, the eigenvalues of $R_{u}$ are the roots of a degree-two polynomial in $\mathbb{Q}[X, \lambda]$ (the degree of the polynomial is two instead of three because $\mathcal{R}$ is a simple ruled quadric); furthermore, the eigenvectors have to be normalized, introducing a new level in the nested square roots; the coefficients of the transformation matrix are thus expressed with 2 levels of nested square roots in $\mathbb{Q}[\lambda]$. Also in Step 2, some other coefficients expressed with 4 levels of nested roots appear in the parameterization $\mathbf{X}$. Indeed, these terms appear in $\mathbf{X}$ as the square roots of the eigenvalues of $R_{u}$, which are solutions of a degree-two polynomial in $\mathbb{Q}[X, \lambda]$.

- In Step 3, a square root appears when solving the degree 2 equation (1.3). Note, however, that this radical is the square root of a polynomial in $u$, the parameter of the curve of intersection.

It is interesting to point out that the solutions produced by Levin's method tend to be very complicated in practice. Consider the simple example where $\mathcal{P}$ is a hyperbolic paraboloid with equation $a x_{1}^{2}+b x_{1} x_{2}-$ $x_{3}+1=0, a, b>0$ and $\mathcal{Q}$ is anything. Then, $\mathcal{R}$ can be chosen equal to $\mathcal{P}$, which substantially simplifies the solution $(\lambda=0$ and the number of levels of nested radical is 2 instead of 4$)$. Even then, the solution $\mathcal{T X}$ has the form

$$
\left(\begin{array}{c}
\frac{b(u+v) / \sqrt{2}}{\sqrt{b^{2}+\left(\sqrt{a^{2}+b^{2}}-a\right)^{2}} \sqrt{\sqrt{a^{2}+b^{2}}+a}}+\frac{b(u-v) / \sqrt{2}}{\sqrt{b^{2}+\left(\sqrt{a^{2}+b^{2}}+a\right)^{2}} \sqrt{\sqrt{a^{2}+b^{2}}-a}} \\
\frac{\left(\sqrt{a^{2}+b^{2}}-a\right)(u+v) / \sqrt{2}}{\sqrt{b^{2}+\left(\sqrt{a^{2}+b^{2}}-a\right)^{2}} \sqrt{\sqrt{a^{2}+b^{2}}+a}}-\frac{\left(\sqrt{a^{2}+b^{2}}+a\right)(u-v) / \sqrt{2}}{\sqrt{b^{2}+\left(\sqrt{a^{2}+b^{2}}+a\right)^{2}} \sqrt{\sqrt{a^{2}+b^{2}}-a}} \\
u v+1 \\
1
\end{array}\right) .
$$

where $v$ still has to be replaced by its solution of (1.3) in terms of $u$. 


\section{Our method}

As in Levin's method, we want to compute an explicit parametric expression for the intersection of two quadric surfaces given by their implicit equations. However, we only report the components of the intersection that are of strictly positive dimension. This choice is consistent with the applications we are interested in, such as CSG-to-B-Rep conversion, because it only means that the primitives defining the CSG models are considered as open volumes. As Theorem 3 will show, not reporting components of dimension zero might simplify the computations.

We improve Levin's method in the following different ways. First we consider the quadric surfaces in the real projective space of dimension three, $\mathbb{P}^{3}$. One of the keys to Levin's method is the existence of parameterizations of "enough" canonical quadric surfaces (the simple ruled quadrics) such that after substitution into the implicit equation of another quadric, we get a second degree equation in one variable with a discriminant $\Delta$ of degree four in another variable. We generalize to projective space that aspect of Levin's method by presenting in Table 1.2 parameterizations, with the above property, for of all quadrics, except those of signature $(3,1)$ (see Theorem 2). (Euclidean quadrics of signature $(3,1)$ are ellipsoids, hyperboloids of two sheets, and elliptic paraboloids.) Considering quadrics in projective space instead of Euclidean space reduces the number of times a quadric $\mathcal{R}$, distinct from $\mathcal{P}, \mathcal{Q}$, has to be searched for in the pencil; indeed, in projective space, a search has to be performed if and only if both $\mathcal{P}$ and $\mathcal{Q}$ are of signature $(3,1)$, although, in Euclidean space, a search has to be performed if and only if both $\mathcal{P}$ and $\mathcal{Q}$ are of signature $(3,1)$ or amongst the cones, elliptic cylinders or hyperboloids of one sheet.

Theorem 2 Let $P^{\prime}$ be a symmetric real $4 \times 4$ matrix. The parameterizations $\mathbf{X}$ presented in Table 1.2 are such that $\mathbf{X}^{T} P^{\prime} \mathbf{X}$ is equal to $a w^{2}+b w t+c t^{2}, a(w t)^{2}+b w t+c$, or $a w^{2}+b w+c$, for the first, second and three last parameterizations respectively, where $\Delta=b^{2}-4 a c$ is a homogeneous polynomial of degree 4 in the variables $u, v$.

Our other key result for avoiding the apparition of irrational numbers is the following:

Theorem 3 If two quadric surfaces $\mathcal{P}$ and $\mathcal{Q}$ both have signature $(3,1)$ and intersect in more than two points (in $\mathbb{P}^{3}$ ) then there exists a rational number $\lambda$ such that $\mathcal{P}-\lambda \mathcal{Q}$ is not of signature $(3,1)$.

This result is of interest because of the following two reasons. First it ensures that the two quadrics, $\mathcal{P}$ and $\mathcal{R}$, we end up intersecting have 
Table 1.2. Parameterization of projective quadric surfaces

\begin{tabular}{|c|c|c|}
\hline $\begin{array}{l}\text { signature } \\
\text { of } \mathcal{Q}\end{array}$ & $\begin{array}{l}\text { canonical equation } \\
\qquad a_{i}>0\end{array}$ & $\begin{array}{c}\text { parameterization } \\
\mathbf{X}=\left[x_{1}, x_{2}, x_{3}, x_{4}\right] \in \mathbb{P}^{3}\end{array}$ \\
\hline$(4,0)$ & $a_{1} x_{1}^{2}+a_{2} x_{2}^{2}+a_{3} x_{3}^{2}+a_{4} x_{4}^{2}=0$ & $\mathcal{Q}=\emptyset$ \\
\hline$(3,1)$ & $a_{1} x_{1}^{2}+a_{2} x_{2}^{2}+a_{3} x_{3}^{2}-a_{4} x_{4}^{2}=0$ & $\operatorname{det} Q<0$ \\
\hline$(3,0)$ & $a_{1} x_{1}^{2}+a_{2} x_{2}^{2}+a_{3} x_{3}^{2}=0$ & $\mathcal{Q}$ is a point \\
\hline$(2,2)$ & $a_{1} x_{1}^{2}+a_{2} x_{2}^{2}-a_{3} x_{3}^{2}-a_{4} x_{4}^{2}=0$ & $\mathbf{X}=\left[\frac{u t+v w}{\sqrt{a_{1}}}, \frac{u w-v t}{\sqrt{a_{2}}}, \frac{u t-v w}{\sqrt{a_{3}}}, \frac{u w+v t}{\sqrt{a_{4}}}\right]$ \\
\hline$(2,1)$ & $a_{1} x_{1}^{2}+a_{2} x_{2}^{2}-a_{3} x_{3}^{2}=0$ & $\mathbf{X}=\left[\frac{u^{2}+v^{2}}{2 \sqrt{a_{1}}}, \frac{u^{2}-v^{2}}{2 \sqrt{a_{2}}}, \frac{u v}{(u, v, w, t) \in \mathbb{P}^{3}}, w t\right]$ \\
\hline$(2,0)$ & $a_{1} x_{1}^{2}+a_{2} x_{2}^{2}=0$ & $\mathbf{X}=[0, \underset{u}{0}, u, v],(u, v) \in \mathbb{P}^{1}$ \\
\hline$(1,1)$ & $a_{1} x_{1}^{2}-a_{2} x_{2}^{2}=0$ & $\begin{aligned} \mathbf{X}=\left[\frac{w}{\sqrt{a_{1}}}, \frac{w}{\sqrt{a_{2}}}, v, w\right] & \\
& \mathbf{X}=\left[\frac{u}{\sqrt{a_{1}}},-\frac{u}{\sqrt{a_{2}}}, v, w\right] \\
(u, v, w) \in \mathbb{P}^{2} & \end{aligned}$ \\
\hline$(1,0)$ & $a_{1} x_{1}^{2}=0$ & $\mathbf{X}=[0, u, v, w],(u, v, w) \in \mathbb{P}^{2}$ \\
\hline
\end{tabular}

rational coefficients. Secondly, the $\lambda \in \mathbb{Q}$, such that $\mathcal{R}(\lambda)=\mathcal{P}-\lambda \mathcal{Q}$ is not of signature $(3,1)$, can be computed, most of the time, with normal floating-point arithmetic. Indeed, first notice that $\mathcal{R}(\lambda)$ is not of signature $(3,1)$ if and only if $\operatorname{det} R(\lambda) \geqslant 0$, and that $\exists \lambda \in \mathbb{R}$ such that $\operatorname{det} R(\lambda) \geqslant 0$, by Theorem 1 . It follows that, in most of the cases, there exists an interval of $\lambda \in \mathbb{R}$ on which $\operatorname{det} R(\lambda) \geqslant 0$. Thus, we can compute approximatively the roots of $\operatorname{det} R(\lambda)=0$, then choose a rational number in each interval induced by these computed roots, and find out whether one of these rational numbers is such that $\operatorname{det} R(\lambda) \geqslant 0$. If we found such a $\lambda$ we report the quadric $\mathcal{R}(\lambda)$ which is not of signature $(3,1)$; due to the lack of space, we do not discuss here how to compute $\lambda$ otherwise.

Our last contribution is the introduction of Gauss' reduction method for quadratic forms for transforming $\mathcal{R}$ into a canonical frame in Step 2. Using Gauss' reduction method instead of finding an orthogonal transformation as in Levin's method, simplifies substantially the form of the solutions. As an example, using just Gauss' reduction method on the example of Section 3 where $\mathcal{P}$ is a hyperbolic paraboloid of equation $a x_{1}^{2}+b x_{1} x_{2}-x_{3}+1=0, a, b>0$, gives a solution $\mathcal{T} \mathbf{X}$ of the form $\left(\frac{v}{\sqrt{a}}, \frac{\sqrt{a}(u-v)}{b}, u v+1,1\right)^{T}$.

Our algorithm is as follows.

1 Find a quadric surface $\mathcal{R}$ with rational coefficients in the pencil generated by $\mathcal{P}$ and $\mathcal{Q}$ such that $\operatorname{det} R \geqslant 0$, or report that the intersection of $\mathcal{P}$ and $\mathcal{Q}$ is of dimension 0 . This is achieved as follows. If 
$\operatorname{det} Q \geqslant 0$ then choose $\mathcal{R}=\mathcal{Q}$. Otherwise, if there exists $\lambda \in \mathbb{Q}$ such that $\operatorname{det}(P-\lambda Q) \geqslant 0$, then compute such a $\lambda$ and set $\mathcal{R}=\mathcal{P}-\lambda \mathcal{Q}$. Otherwise, report that $\mathcal{P} \cap \mathcal{Q}$ is of dimension 0 (see Theorem 3).

2 If $\mathcal{R}$ has signature $(4,0)$ or $(3,0)$ then report that $\mathcal{P} \cap \mathcal{Q}$ is of dimension 0 (see Table 1.2). Otherwise, using Gauss' method for reducing quadratic forms into diagonal forms [10], compute a (non-orthogonal) transformation $\mathcal{T}$ which sends $R$ into canonical form. In that coordinate frame, in which $\mathcal{T}^{T} R \mathcal{T}$ is diagonal, the quadric surface $\mathcal{R}$ has one of the parameterizations $\mathbf{X}$ of Table 1.2 (by Sylvester's Inertia Law, the signatures of $R$ and $\mathcal{T}^{T} R \mathcal{T}$ are equal). Compute the matrix $P^{\prime}=\mathcal{T}^{T} P \mathcal{T}$ of the quadric surface $\mathcal{P}$ in that coordinate frame and consider the equation $\mathbf{X}^{T} P^{\prime} \mathbf{X}=0$.

3 Solve $\mathbf{X}^{T} P^{\prime} \mathbf{X}=0$ as an equation of degree two according to Theorem 2 , and determine the domains of $(u, v) \in \mathbb{P}^{1}$ on which the solutions are defined. Substitute, depending on the case, $(w, t), w t$ or $w$ in terms of $(u, v)$ in $\mathbf{X}$. (If $a, b, c$ vanish simultaneously for some values of $(u, v)$, then replace $(u, v)$ by those values in $\mathbf{X}$ and let $(w, t) \in \mathbb{P}^{1}$.) We get a parameterization of $\mathcal{P} \cap \mathcal{Q}=\mathcal{P} \cap \mathcal{R}$ in the coordinate system where $\mathcal{R}$ is canonical.

4 Report $\mathcal{T} \mathbf{X}(u, v)$ and the corresponding domain of $(u, v) \in \mathbb{P}^{1}$ on which the solution is defined, as the parameterized equation of $\mathcal{P} \cap \mathcal{Q}$ in the global coordinate frame.

The algorithm we presented here produces an explicit parametric representation for the intersection of two quadric surfaces $\mathcal{P}$ and $\mathcal{Q}$ given by their implicit equations (with rational coefficients), such that all the coefficients are in $\mathbb{Q}\left[\sqrt{a_{1}}, \sqrt{a_{2}}, \sqrt{a_{3}}, \sqrt{a_{4}}\right]$ where the $a_{i}$ are the coefficients of $\mathcal{R}$ in canonical form through $\mathcal{T}$ (i.e., the coefficients on the diagonal of $\left.\mathcal{T}^{T} R \mathcal{T}\right)$.

\section{Notes}

1. Levin's method fails if $\operatorname{det}\left(R_{u}(\lambda)\right) \equiv 0$ because, then, the type of all the quadrics $\mathcal{R}(\lambda), \lambda \in \mathbb{R}$, have to be computed. However, the method can be easily fixed by considering the roots of other polynomials, such as $\operatorname{det}(R(\lambda))$, that naturally appear when considering the classification of quadric surfaces as in [12].

\section{References}

[1] M. Benouamer, D. Michelucci, and B. Peroche. Error-free boundary evaluation based on a lazy rational arithmetic: a detailed implementation. Computer-Aided Design, 26(6):403-416, 1994.

[2] A. Bowyer, J. Berchtold, D. Eisenthal, I. Voiculescu, and K. Wise. Interval methods in geometric modeling. In Proc. of International 
Conference on Geometric Modeling and Processing, Hong Kong, 2000. Invited presentation.

[3] R. Farouki, C. Neff, and M. O'Connor. Automatic parsing of degenerate quadric-surface intersections. ACM Transactions on Graphics, 8(3):174-203, 1989.

[4] S. Fortune. Polyhedral modelling with exact arithmetic. In Proc. of ACM Symposium on Solid Modeling and Applications, pages 225$234,1995$.

[5] N. Geismann, M. Hemmer, and E. Schoemer. Computing the intersection of quadrics: Exactly and actually. In Proc. of ACM Symposium on Computational Geometry, 2001. To appear.

[6] R. Goldman and J. Miller. Combining algebraic rigor with geometric robustness for the detection and calculation of conic sections in the intersection of two natural quadric surfaces. In Proc. of ACM Symposium on Solid modeling foundations and CAD/CAM applications, pages 221-231, 1991.

[7] C.-K. Hung and D. Ierardi. Constructing convex hulls of quadratic surface patches. In Proceedings of 7 th CCCG (Canadian Conference on Computational Geometry), Quebec, Canada, pages 255-260, 1995.

[8] J. Keyser, S. Krishnan, and D. Manocha. Efficient and accurate Brep generation of low degree sculptured solids using exact arithemtic: I - Representations. Computer Aided Geometric Design, 16(9):841859, 1999.

[9] J. Keyser, S. Krishnan, and D. Manocha. Efficient and accurate Brep generation of low degree sculptured solids using exact arithemtic: II - Computation. Computer Aided Geometric Design, 16(9):861$882,1999$.

[10] T. Lam. The Algebraic Theory of Quadratic Forms. W.A. Benjamin, Reading, MA, 1973.

[11] J. Levin. A parametric algorithm for drawing pictures of solid objects composed of quadric surfaces. Communications of the ACM, 19(10):555-563, 1976.

[12] J. Levin. Mathematical models for determining the intersections of quadric surfaces. Computer Graphics and Image Processing, 11(1):73-87, 1979.

[13] R. Sarraga. Algebraic methods for intersections of quadric surfaces in GMSOLID. Computer Vision, Graphics, and Image Processing, 22:222-238, 1983.

[14] K. Sugihara. How to make geometric algorithms robust. IEICE Transactions on Information and Systems, E83-D(3):447-454, 2000. 